\title{
Cigarette smoke modulates rhinovirus- induced airway epithelial cell chemokine production
}

\author{
M.H. Hudy, S.L. Traves, S. Wiehler and D. Proud
}

ABSTRACT: Human rhinovirus (HRV) infections induce epithelial cell production of chemokines that may contribute to the pathogenesis of exacerbations of chronic obstructive pulmonary disease (COPD) and asthma. Cigarette smoking is the predominant risk factor for the development of COPD and also aggravates asthma symptoms. We examined whether cigarette smoke extract (CSE) modulates viral inflammation by altering the profile of HRV-induced epithelial chemokine production.

Purified HRV-16, and CSE were used to examine the effects on CXC chemokine ligand (CXCL)8 and CXCL10 production from both primary human bronchial epithelial cells and the BEAS-2B epithelial cell line.

Both CSE and HRV-16 induced CXCL8 production and, when used in combination, induced at least an additive production of CXCL8 compared with either stimulus alone. In contrast, CSE did not induce CXCL10 and markedly inhibited HRV-16-induced CXCL10 production. Inhibition of HRV-16-induced CXCL10 by CSE was mediated, at least in part, via transcriptional regulation. The increased CXCL8 production seen with the combination of CSE and HRV-16 was not due to transcriptional regulation but was associated with CXCL8 mRNA stabilisation.

Thus, CSE differentially modulates HRV-16-induced chemokine production from human airway epithelial cells in a manner that might be expected to alter inflammatory cell profiles.

KEYWORDS: Airway epithelium, chemokines, cigarette smoke, host defence, human rhinovirus, inflammation

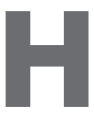
uman rhinovirus (HRV) infections are a major cause of exacerbations of both chronic obstructive pulmonary disease (COPD) and asthma [1]. Although the mechanisms by which HRV infections induce such exacerbations of lower airway diseases are not fully delineated, it is thought that infection of airway epithelial cells trigger epithelial responses that contribute to increased airway inflammation. In support of this, epithelial cells infected with HRV produce numerous chemokines, cytokines and host defence molecules [2]. Several of these chemokines, including CXCL8 and CXCL10, are also observed in increased amounts in airway secretions during in vivo HRV infections. Levels of both CXCL8 and CXCL10 correlate with symptom severity during HRV infections [3, 4] CXCL8 is a potent chemoattractant for neutrophils, and neutrophil numbers in airway secretions have been shown to correlate with disease severity in COPD and during viral exacerbations of asthma [5,6]. CXCL10 is a chemoattractant for activated lymphocytes and natural killer cells that have been linked to host antiviral defences.

Cigarette smoke has profound effects on airway biology and is the major risk factor for the development of COPD [7]. In addition, some $25 \%$ of asthmatic subjects smoke [8], and these individuals have worse respiratory symptoms, more frequent hospitalisations and decreased quality of life, compared with asthmatic subjects who do not smoke [9]. Moreover, asthmatic subjects who smoke are also less responsive to corticosteroid treatment than nonsmoking asthmatics [10].

Interestingly, it has been reported that both the frequency and severity of viral infections is greater in cigarette smokers compared with nonsmokers [11, 12]. Although a few studies

\section{AFFLLIATIONS}

Airway Inflammation Group, Institute of Infection, Immunity and Inflammation and Dept of Physiology and Pharmacology, University of

Calgary, Calgary, AB, Canada.

\section{CORRESPONDENCE}

D. Proud

Dept of Physiology \& Pharmacology, HRIC 4AC60, University of Calgary

3330 Hospital Drive NW

Calgary

$\mathrm{AB}$

T2N 4N1

Canada

E-mail: dproud@ucalgary.ca

Received:

Aug 132009

Accepted after revision:

Sept 302009

First published online:

Oct 202009 
have investigated the combined effects of viruses and cigarette smoke, to date, there have been no studies looking at the effects of cigarette smoke on epithelial responses to HRV infection. The current studies were performed to determine if, and how, cigarette smoke modulates HRV-induced chemokine production from human airway epithelial cells.

\section{MATERIALS AND METHODS Materials}

The following reagents were purchased from the indicated suppliers: bronchial epithelial cell basal medium (BEBM) and additives to create serum-free bronchial epithelial cell growth medium (BEGM; Lonza, Walkersville, MD, USA); Hank's balanced salt solution (HBSS), TRIzol reagent, and fetal bovine serum (FBS; Invitrogen, Burlington, ON, Canada); DNase I (Ambion, Austin, TX, USA); TaqMan Master Mix, 20X glyceraldehyde-3-phosphate dehydrogenase (GAPDH), RNase inhibitor and reverse transcriptase (Applied Biosystems, Foster City, CA, USA); the firefly luciferase reporter plasmid pGL4 basic and passive lysis buffer (Promega, Madison, WI, USA); firefly luciferase assay kit (Biotium Inc., Hayward, CA); the selective p38 mitogen-activated protein kinase (MAPK) inhibitor, SB203580 (4-(4-fluorophenyl)-2-(4-methylsulfinylphenyl)-5(4-pyridyl) 1H-imadazole; Calbiochem, Gibbstown, NJ, USA); transIT-LT1 transfection reagent (Mirus, Madison, WI); antibodies against phospho-p38 and total-p38 (Cell Signalling Technology, Beverly, MA, USA). All other chemicals were purchased from Sigma-Aldrich (Oakville, ON, Canada).

\section{Virus and cell lines}

The BEAS-2B cell line was a gift from C. Harris (National Cancer Institute, Bethesda, MD, USA). HRV type 16 (HRV-16), HRV type 1A (HRV-1A), H1-HeLa cells and WI-38 cells were purchased from the American Type Culture Collection (Manassas, VA, USA). HRV-16 viral stocks were propagated in WI-38 cells, while HRV-1A stocks were propagated in H1HeLa cells. Each viral stock was purified to remove soluble products of the propagating cells by sucrose density centrifugation, as previously described [13]. Viral titres were also determined 5 days after inoculation of monolayers of WI-38 or H1-HeLa cells, as previously described [13].

\section{Epithelial cell culture}

Normal human lungs that were not used for transplant were obtained from a tissue retrieval service (International Institute for the Advancement of Medicine, Edison, NJ, USA). Ethical approval to receive tissues was obtained from both the Conjoint Health Research Ethics Board of the University of Calgary (Calgary, AB, Canada) and from the Internal Ethics Board of the International Institute for the Advancement of Medicine (Edison, NJ, USA). All donors had negative serologies for HIV-1/2, human T-lymphotropic virus (HTLV)-1/2, hepatitis A, B and C, and syphilis. In the current study, lungs from 10 different donors (nine male, age range 1856 yrs old) were used. Of these, six subjects died of cerebral vascular events and four from head trauma. Lungs were received within 24-36 h after surgical removal. Primary human bronchial epithelial (HBE) cells were obtained by protease digestion of dissected airways, as previously described [14]. HBE and BEAS-2B cells were grown in submersion culture in BEGM and incubated at $37^{\circ} \mathrm{C}$ in $5 \%$
$\mathrm{CO}_{2}$. Primary cell cultures achieved confluence in 2 weeks and the epithelial nature of cells was confirmed by cytokeratin staining [14]. Prior to stimulation, cells were cultured overnight in BEGM from which hydrocortisone was removed, and this medium was used for all experiments.

\section{Preparation of cigarette smoke extract}

Cigarette smoke extract (CSE) was freshly prepared by a minor modification of previously published methods [15]. In brief, crude CSE was generated by bubbling one research grade cigarette (3R4F, College of Agriculture Reference Cigarette Program, University of Kentucky, Lexington, KY, USA) per $4 \mathrm{~mL}$ of BEGM without hydrocortisone at a rate of $5 \mathrm{~min}$ per cigarette using a syringe apparatus. The crude CSE was filtered through a $0.22 \mu \mathrm{m}$ filter and subsequently adjusted with medium to an absorbance of 0.15 at $320 \mathrm{~nm}$. This solution was taken as $100 \%$ CSE. For experiments using aged CSE, the extract was prepared as described and left at $4^{\circ} \mathrm{C}$ for $24 \mathrm{~h}$.

\section{Viral infection and stimulation of epithelial cells}

BEAS-2B cells were infected with HRV-16 at $10^{4.5} 50 \%$ tissue culture-infective dose (TCID50) $\mathrm{U} \cdot \mathrm{mL}^{-1}$ (multiplicity of infection (MOI) of $\sim 0.1$ ), while HBE were infected with $10^{5.5}$ TCID50 $\mathrm{U} \cdot \mathrm{mL}^{-1}(\mathrm{MOI} \sim 1.0)$. The higher dose used in HBE is needed to ensure robust responses, as it has been shown that, even at high doses of HRV, no more than $10 \%$ of HBE are infected [16]. Infections were performed in the presence or absence of CSE. All experiments were performed using 50\% CSE unless stated otherwise. Cells were incubated at $34^{\circ} \mathrm{C}$ in $5 \% \mathrm{CO}_{2}$. Viability of cells exposed to HRV-16, CSE or the combination was determined using both 3-(4,5-dimethylthiazol-2-yl)-2,5-diphenyl tetrazolium bromide (MTT) and lactate dehydrogenase (LDH) assays. BEAS-2B cells were pre-incubated with the selective p38 mitogen-activated MAPK inhibitor, SB203580 $(3 \mu \mathrm{M})$, or vehicle control (DMSO) for $1 \mathrm{~h}$ at $37^{\circ} \mathrm{C}$ before stimulation with HRV-16 and/or CSE.

\section{Transfection of epithelial cells with polyinosinic:polycytidylic acid}

Polyinosinic:polycytidylic acid (poly(I:C)) $(0.1 \mu \mathrm{g})$ was transfected into cells in BEBM with no additives using TransIT. After $1 \mathrm{~h}$, cells were washed with HBSS and cultured in medium with or without CSE for $24 \mathrm{~h}$.

\section{Real-time RT-PCR}

CXCL8 and CXCL10 mRNA expression analysis was performed by real-time RT-PCR. For each sample, $400 \mathrm{ng}$ of input RNA was reverse transcribed into complementary DNA (cDNA), followed by PCR amplification in the presence of specific primers and probes for the gene of interest or the housekeeping gene GAPDH. Primer and probe sequences for CXCL8 and CXCL10 have been described previously [4, 17]. In each case, a synthetic first-strand cDNA amplicon was used to generate standard curves to permit absolute quantification of mRNA. Data were expressed as femtograms or attograms calculated from the standard curve after correction for minor variations in GAPDH levels.

\section{CXCL8 mRNA stability studies}

BEAS-2B cells were exposed to HRV-16, CSE or the combination for $2 \mathrm{~h}$ and actinomycin $\mathrm{D}$ was added at a final 
concentration of $10 \mu \mathrm{g} \cdot \mathrm{mL}^{-1}$. Cellular RNA was then isolated at various times for analysis of CXCL8 mRNA levels.

\section{ELISAS}

CXCL8 and CXCL10 were assayed by ELISA using matched antibody pairs according to the manufacturer's protocol (R\&D Systems, Minneapolis, MN, USA).

\section{CXCL8 and CXCL10 promoter constructs}

The 972-bp CXCL10 promoter construct has been previously described [4]. A 720-bp CXCL8 promoter construct, corresponding to the sequence from -712 to +8 (relative to the transcriptional start site) of the 5'-flanking region of the human CXCL8 gene, was generated from genomic DNA using forward 5'-CGGGGTACCTATAGTCAGTCCTTACATTGC-3' and reverse 5'-CCCAAGCTTCTTATGGAGTGCTCCGGT GGC-3' primers incorporating the KpnI and HindIII restriction sites (underlined) used for insertion into the reporter plasmid. Both promoters were cloned upstream of the inducible firefly luciferase gene in pGL4 basic.

\section{Transient transfection and luciferase assay}

The CXCL8 $(0.2 \mu \mathrm{g})$ or the CXCL10 promoter construct $(1 \mu \mathrm{g})$ were transfected into sub confluent $(40-50 \%)$ monolayers of BEAS-2B cells, as previously described [17]. Cells were washed, stimulated with HRV-16, CSE or the combination for either $5 \mathrm{~h}$ (CXCL8) or $24 \mathrm{~h}$ (CXCL10) and luciferase activity was measured as described [17].

\section{Western blotting for p38 MAPK activation}

Cell extraction, sodium dodecyl sulphate polyacrylamide gel electrophoresis (SDS-PAGE) and probing with the phosphop38 MAPK and total-p38 MAPK antibody (Ab) were performed, as previously described [17].

\section{Statistical analysis}

Normally distributed data were analysed using either paired t-tests or one-way ANOVA with student Newman-Keuls post hoc analysis. Data that were not normally distributed were analysed using Kruskal-Wallis ANOVA followed by Wilcoxon matched-pairs signed-rank test. To determine whether there was synergy between HRV-16 and CSE, the sum of HRV-16 alone and CSE alone was compared with HRV-16+CSE. Paired t-tests or Wilcoxon matched-pairs signed-rank tests were used to determine differences. Data that had two independent variables were analysed using twoway repeated measures ANOVA with Bonferroni's multiple comparison post hoc analysis. Values of $\mathrm{p}<0.05$ were considered significant.

\section{RESULTS}

\section{CSE does not affect cell viability or viral titre}

Concentrations of $50 \%$ CSE or lower alone or in combination with HRV-16 did not affect viability of HBE or BEAS-2B cells as assessed using both MTT (see fig. A in the supplementary material) and LDH assays (data not shown). To determine effects of CSE on viral replication, cells were exposed for $1 \mathrm{~h}$ to HRV alone or HRV+CSE for $1 \mathrm{~h}$. Cells were washed and fresh medium, with or without CSE, was added. Viral titres in supernatants obtained after $24 \mathrm{~h}$, expressed as Log TCID50, were not different between cells exposed to HRV-16 alone
$(6.0 \pm 0.04)$ and HRV-16 plus CSE $(5.9 \pm 0.35)$. Values are mean \pm SEM of three separate experiments (see fig. $B$ in the supplementary material).

\section{CSE and HRV-16 induce CXCL8 production}

Based on preliminary time course studies (see fig. $C$ in the supplementary material) CXCL8 mRNA levels were measured at $5 \mathrm{~h}$ post stimulation, while protein levels were assessed at $24 \mathrm{~h}$ after stimulation. HRV-16 alone and CSE each induced CXCL8 mRNA and protein from both HBE (fig. 1a and b) and BEAS-2B cells (fig. 1c and d). The combination of HRV-16 and CSE induced levels of CXCL8 mRNA and protein that were significantly greater than those induced by either stimulus alone. CXCL8 mRNA and protein induced by the combination of CSE and HRV-16 in BEAS-2B cells was synergistic compared with the two stimuli individually (fig. 1c and d). Synergistic induction of CXCL8 mRNA by HRV-16+CSE was also observed in HBE (fig. 1a). By contrast, CXCL8 protein
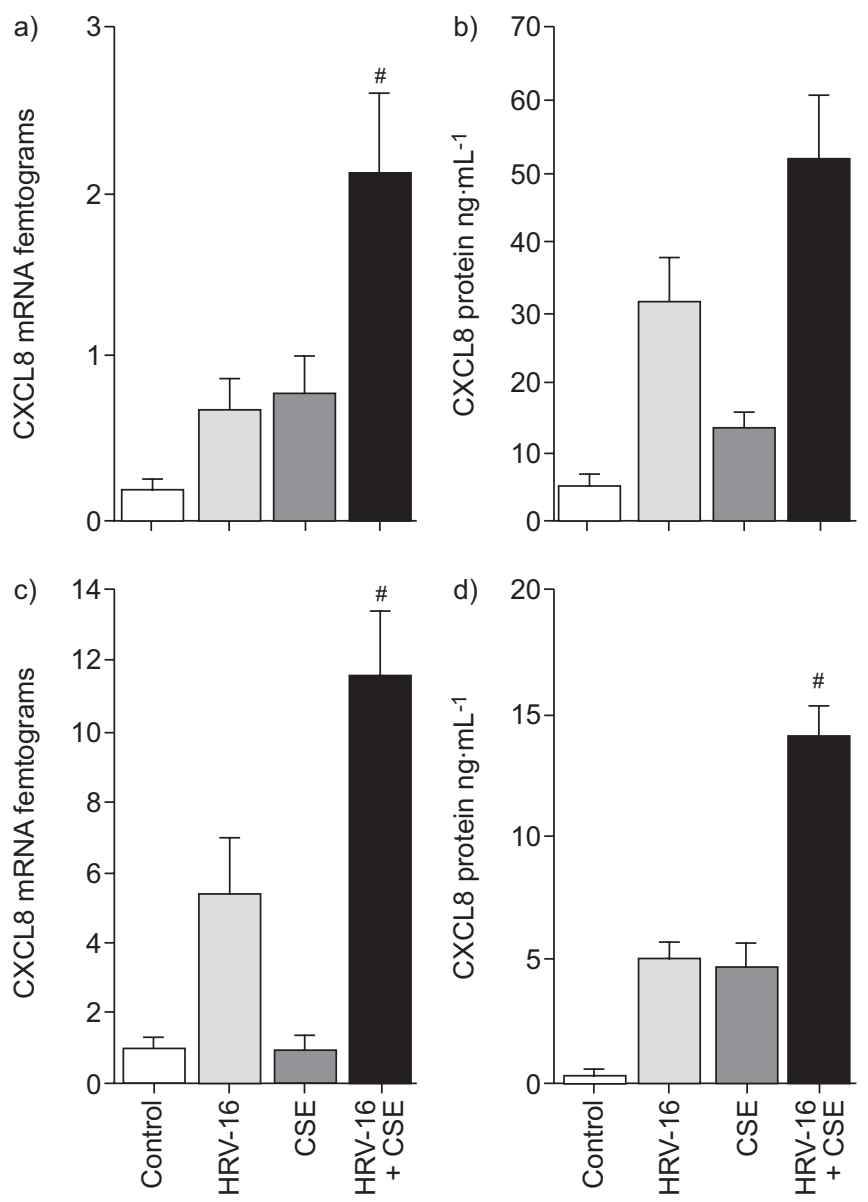

FIGURE 1. Cigarette smoke extract (CSE) and human rhinovirus (HRV)-16 induce $\mathrm{CXC}$ chemokine ligand (CXCL) 8 alone and at least additively increase CXCL8 in combination in airway epithelial cells. a) CXCL8 MRNA levels were determined at $5 \mathrm{~h}$ in human bronchial epithelial (HBE; $\mathrm{n}=10$ ) cells. b) CXCL8 protein levels were determined at $24 \mathrm{~h}$ in HBE cells $(n=9)$. c) CXCL8 mRNA levels were determined at $5 \mathrm{~h}$ in BEAS-2B cells $(n=6)$. d) CXCL8 protein levels were determined at $24 \mathrm{~h}$ in BEAS-2B cells $(n=10) .{ }^{*}$ : significant difference between HRV$16+C S E$ and the sum of values from each treatment alone. Values are mean \pm SEM. 

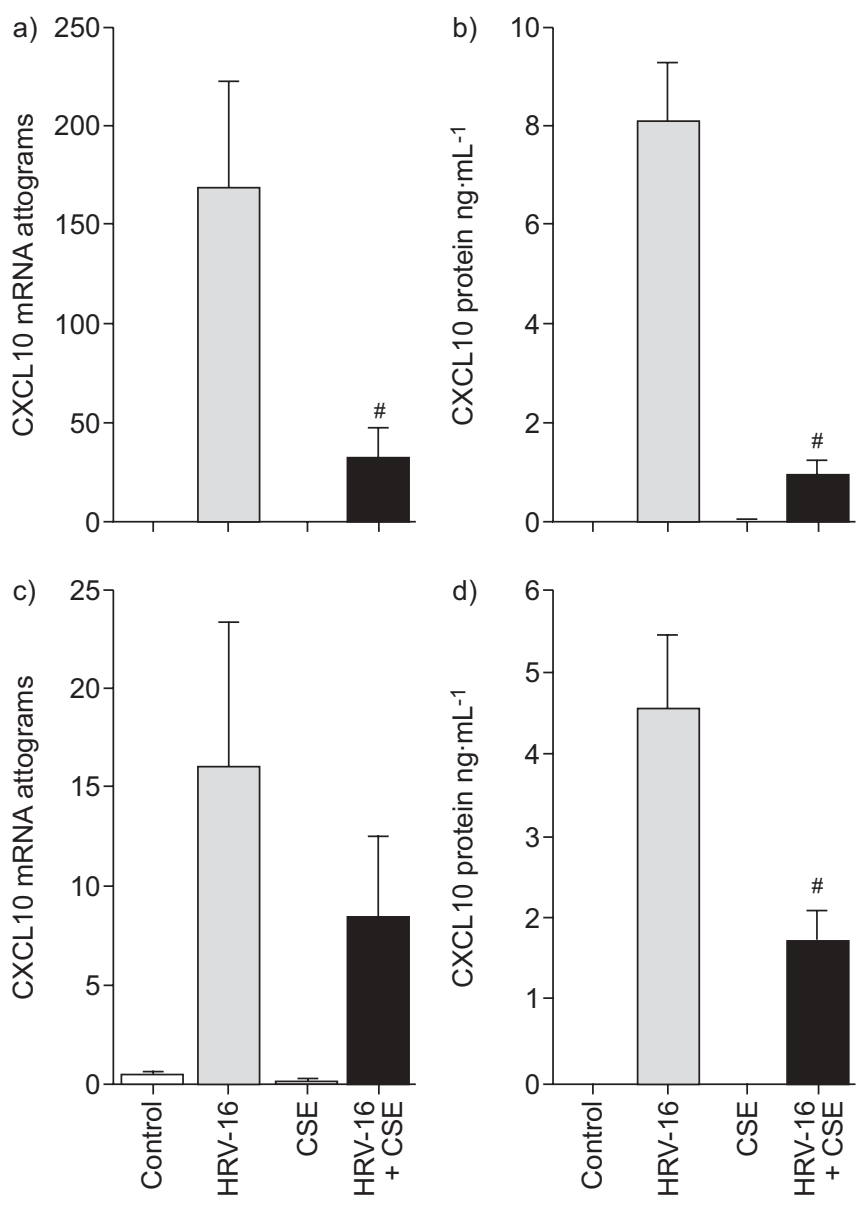

FIGURE 2. Cigarette smoke extract (CSE) inhibits human rhinovirus (HRV)-16induced CXC chemokine ligand (CXCL)10 in airway epithelial cells. a) CXCL10 mRNA $(n=10)$ and b) CXCL10 protein $(n=10)$ levels were determined at $24 \mathrm{~h}$ in human bronchial epithelial (HBE) cells. c) CXCL10 mRNA ( $n=9)$ and d) CXCL10 protein $(n=10)$ levels were determined at $24 \mathrm{~h}$ in BEAS-2B cells. ${ }^{*}$ : significant inhibition with HRV-16+CSE compared with HRV-16 alone. Values are mean \pm SEM.

production from HBE by CSE+HRV-16 was not significantly greater than the sum of responses to each stimulus individually (fig. 1b). The effects of CSE alone or in combination with HRV-16 on CXCL8 protein production was concentration dependent (see fig. D in the supplementary material), but synergistic induction was only observed at 50\% CSE.

\section{CSE inhibits HRV-16-induced CXCL10 expression}

HRV-16 induced CXCL10 mRNA and protein expression in HBE and BEAS-2B cells (fig. 2). By contrast, CSE alone did not induce production of CXCL10 from either cell type, and CSE markedly inhibited HRV-16-induced CXCL10 mRNA and protein expression. In HBE, both HRV-16-induced CXCL10 mRNA and protein were significantly inhibited (fig. 2a and b). In BEAS-2B cells, HRV-16 induced CXCL10 protein production was also significantly inhibited (fig. 2d). The CSE-mediated inhibition of HRV-16 induced CXCL10 mRNA expression in BEAS-2B cells (fig. 2c) just failed to achieve statistical significance $(p=0.054)$. The inhibition of HRV-16-induced CXCL10 by CSE was concentration dependent, with concentrations of
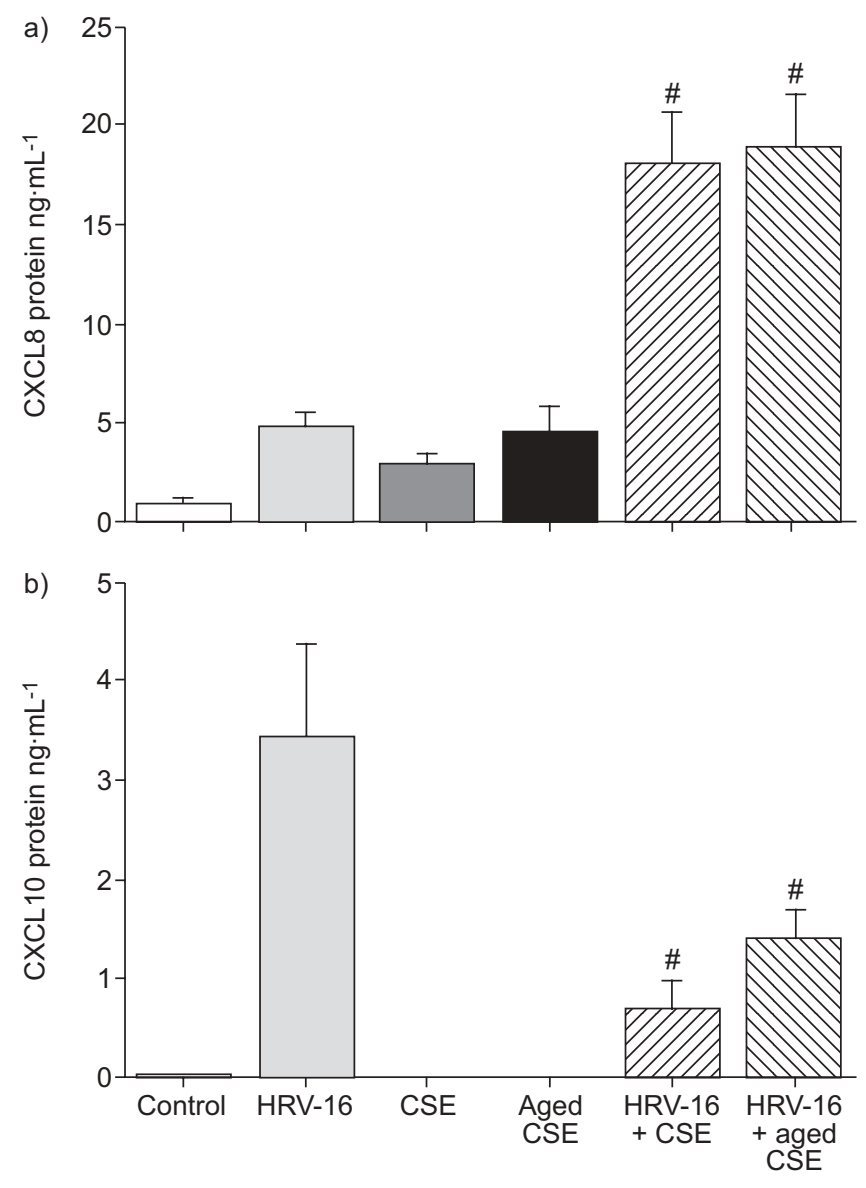

FIGURE 3. The effects of freshly prepared versus aged cigarette smoke extract (CSE) alone or in combination with human rhinovirus (HRV)-16 on CXC chemokine ligand (CXCL) 8 and CXCL10 production in BEAS-2B cells. a) CXCL8 $(n=8)$ and b) CXCL10 $(n=8)$ protein levels were determined at $24 \mathrm{~h}$. ${ }^{*}$ : significant difference between HRV-16+CSE or HRV-16+aged CSE and HRV-16 alone. Values are mean \pm SEM.

CSE as low as $0.005 \%$ still significantly inhibiting HRV-16induced CXCL10 production (see fig. E in the supplementary material).

\section{CSE also modulates chemokine production induced by HRV-1A}

To determine if the effects of CSE on viral production of epithelial chemokines was unique for HRV-16, we also used the minor group rhinovirus serotype, HRV-1A, as a stimulus in BEAS-2B cells. CSE also induced additive induction of CXCL8 when combined with HRV-1A. As was the case with HRV-16, CSE inhibited HRV-1A-induced production of CXCL10 (see fig. $\mathrm{F}$ in the supplementary material).

\section{Induction of CXCL8 and inhibition of CXCL10 is unaffected by aging CSE}

Both freshly prepared CSE and aged CSE induced comparable levels of CXCL8 protein, either alone or in the combination with HRV-16 (fig. 3a). Both fresh CSE or aged CSE when used in combination with HRV-16 synergistically induced CXCL8. 

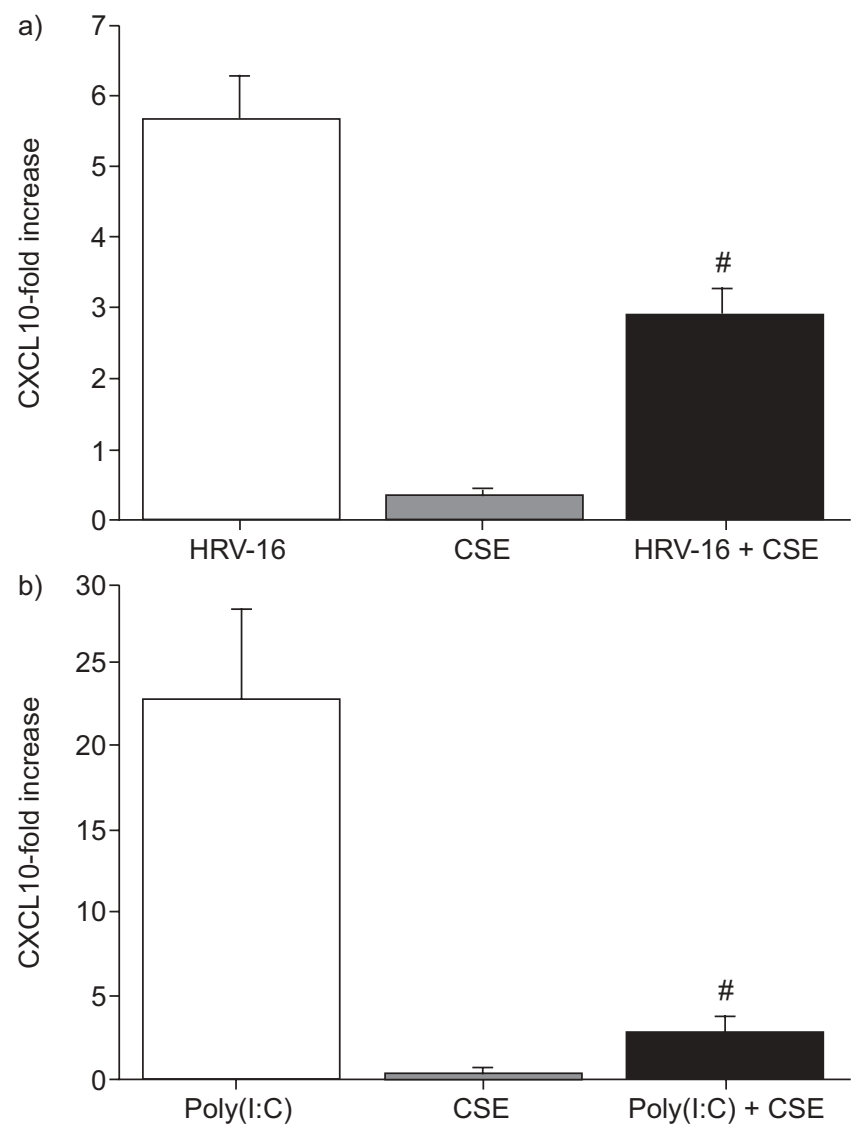

FIGURE 4. Cigarette smoke extract (CSE) inhibits both human rhinovirus (HRV)-16-induced and polyinosinic:polycytidylic acid (poly(l:C))-induced CXC chemokine ligand $(\mathrm{CXCL}) 10$ promoter activation in BEAS-2B cells. a) Cells stimulated with HRV-16, CSE or the combination $(n=14)$. b) Cells were transfected with poly $(l: C)$ in the presence or absence of CSE $(n=6) .{ }^{*}$ : significant inhibition with HRV-16+CSE compared with HRV-16 alone or with poly(l:C)+CSE compared with poly(l:C) alone. Values are mean \pm SEM.

In addition, both freshly prepared CSE and aged CSE significantly inhibited HRV-16-induced CXCL10 to a comparable extent (fig. 3b).

\section{CSE inhibits HRV-16-induced and poly(I:C)-induced CXCL10 promoter activation}

To determine if CSE modulated HRV-16-induced CXCL10 transcription, promoter-luciferase construct studies were performed. As expected, HRV-16 induced CXCL10 promoter activity (fig. 4a). CSE alone did not induce promoter activation and, indeed, significantly suppressed basal promoter drive. In addition, CSE significantly inhibited HRV-16 induced CXCL10 promoter activation (fig. 4a).

We have previously shown that HRV-induced epithelial production of CXCL10 is dependent upon viral replication, and that synthetic double-stranded RNA (dsRNA; poly(I:C)), a mimic for viral dsRNA generated during the replication cycle, also induces CXCL10 production [4, 18]. Poly(I:C) caused robust production of CXCL10 protein in both HBE and BEAS2B cells and, in both cell types, CSE significantly inhibited these responses (see fig. $\mathrm{G}$ in the supplementary material). CSE

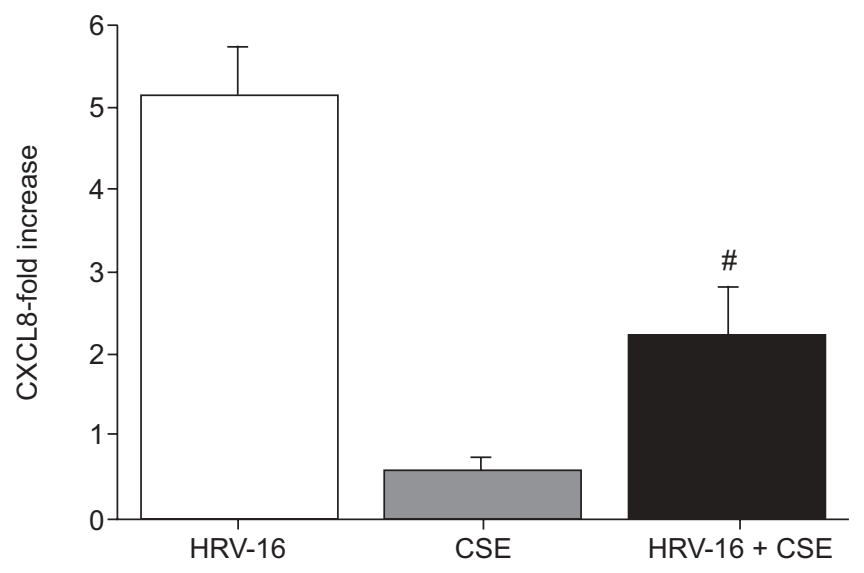

FIGURE 5. Cigarette smoke extract (CSE) in combination with human rhinovirus (HRV)-16 does not enhance CXC chemokine ligand (CXCL)8 promoter activation in BEAS-2B cells. Cells were stimulated with HRV-16, CSE or the combination. *: significant inhibition with HRV-16+CSE compared with HRV-16 alone. Values are mean $\pm \operatorname{SEM}(n=6)$.

also significantly inhibited CXCL10 promoter activation induced by poly(I:C) (fig. 4b).

CSE alone does not induce CXCL8 promoter activation or enhance HRV-16-induced CXCL8 promoter activation

As expected, HRV-16 alone activated the CXCL8 promoter. Surprisingly, not only did CSE alone not induce CXCL8 promoter activation, but it significantly inhibited HRV-16induced promoter drive (fig. 5).

\section{The combination of CSE and HRV-16 increases stability of CXCL8 IRNA}

Preliminary data demonstrated that synergistic induction of CXCL8 steady state mRNA was first observed $2 \mathrm{~h}$ after stimulation (data not shown). Thus, we used a $2 \mathrm{~h}$ exposure before the addition of actinomycin D. In cells treated with either CSE alone or HRV-16 alone, CXCL8 mRNA decayed, with $50 \%$ loss occurring between 2 and $3 \mathrm{~h}$ in each case (fig. 6). However, in cells exposed to the combination of CSE and HRV-16, a significant stabilisation of CXCL8 mRNA was observed, such that there was no significant degradation of CXCL8 mRNA over the $3 \mathrm{~h}$ time period studied (fig. 6).

The effects of CSE alone or in combination with HRV-16 on the p38 MAPK pathway

Inhibition of the 38 MAPK pathway reduces HRV-16 induced CXCL8 production from epithelial cells [17], and the p38 MAPK pathway has been linked to stabilisation of CXCL8 mRNA [19]. In agreement with our earlier studies, HRV-16 induced rapid phosphorylation of p38 MAPK [17]. Consistent with an earlier report [20], CSE also modestly induced phosphorylation of p38 MAPK in BEAS-2B cells. The combination of HRV-16 and CSE induced additive phosphorylation of p38 MAPK at $30 \mathrm{~min}$ (as assessed by densitometry), but the response to the combination was less than additive at later time points (fig. 7a). The selective p38 MAPK inhibitor, SB203580, significantly inhibited CSE-induced CXCL8 protein production as well as CXCL8 production in response to the combination of HRV-16 and CSE (fig. 7b). 


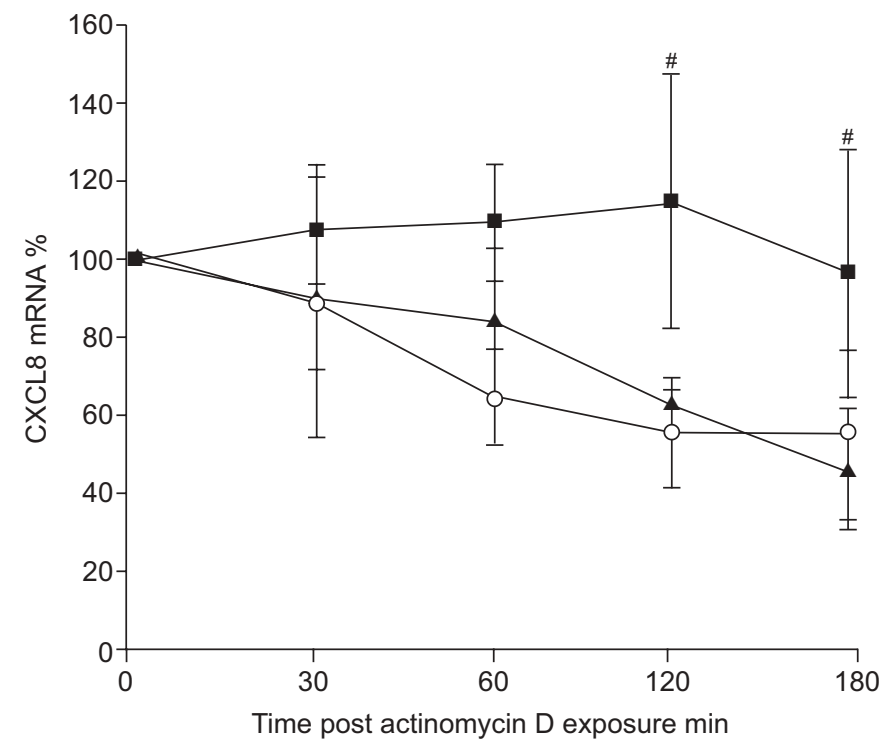

FIGURE 6. The combination of human rhinovirus (HRV)-16 and cigarette smoke extract (CSE) leads to stabilsation of CXC chemokine ligand (CXCL)8 mRNA in BEAS2B cells. Cells were stimulated for $2 \mathrm{~h}$ with HRV-16 $(\mathbf{\Lambda})$, CSE $(O)$ or the combination (匹), and then actinomycin $\mathrm{D}\left(10 \mu \mathrm{g} \cdot \mathrm{mL}^{-1}\right)$ was added. Total cellular RNA was extracted at the times shown and CXCL8 mRNA levels assayed. Data are expressed as a percentage of CXCL8 mRNA at time 0 for each stimulus. ${ }^{*}$ : significant difference between HRV-16+CSE and HRV alone. Values are mean \pm SEM $(n=4)$.

\section{DISCUSSION}

We provide clear evidence that CSE modulates epithelial responses to HRV-16 infection. Although it has been previously reported that CSE itself induces CXCL8 production from epithelial cells [21], our data are the first to show more than additive induction by the combination of CSE and HRV infection. Indeed, synergistic induction was seen for mRNA and protein production from BEAS-2B cells and mRNA from HBE. Protein release from HBE failed to achieve significance for synergy, probably due to the wide range of CXCL8 protein production seen between individual primary HBE donors. By contrast, CSE did not induce CXCL10 production from epithelial cells and strikingly inhibited HRV-induced CXCL10 production. These interactions were not unique for HRV-16, as CSE caused similar modulation of chemokine responses induced by the minor group serotype, HRV-1A. If similar responses occur in vivo, the increases in CXCL8 would be expected to favour a neutrophilic response in the airways and might lead to worse clinical outcomes, given that airway neutrophil numbers correlate with disease severity in both COPD and during viral exacerbations of asthma $[5,6]$. Similarly, if reduced production of CXCL10 occurs in response to cigarette smoke in vivo, this could also worsen clinical outcomes. CXCL10 is a selective chemoattractant for type 1 lymphocytes and natural killer cells that play a role in antiviral defences, and CXCL10 levels are induced during viral exacerbations of asthma [22], possibly as part of the host antiviral defence. Although antinflammatory drugs, such as corticosteroids [23], and host defence molecules, such as nitric oxide [18], suppress HRV-induced epithelial production of CXCL10, these treatments also inhibit a range of other cytokines, including CXCL8. Thus, the differential effects of
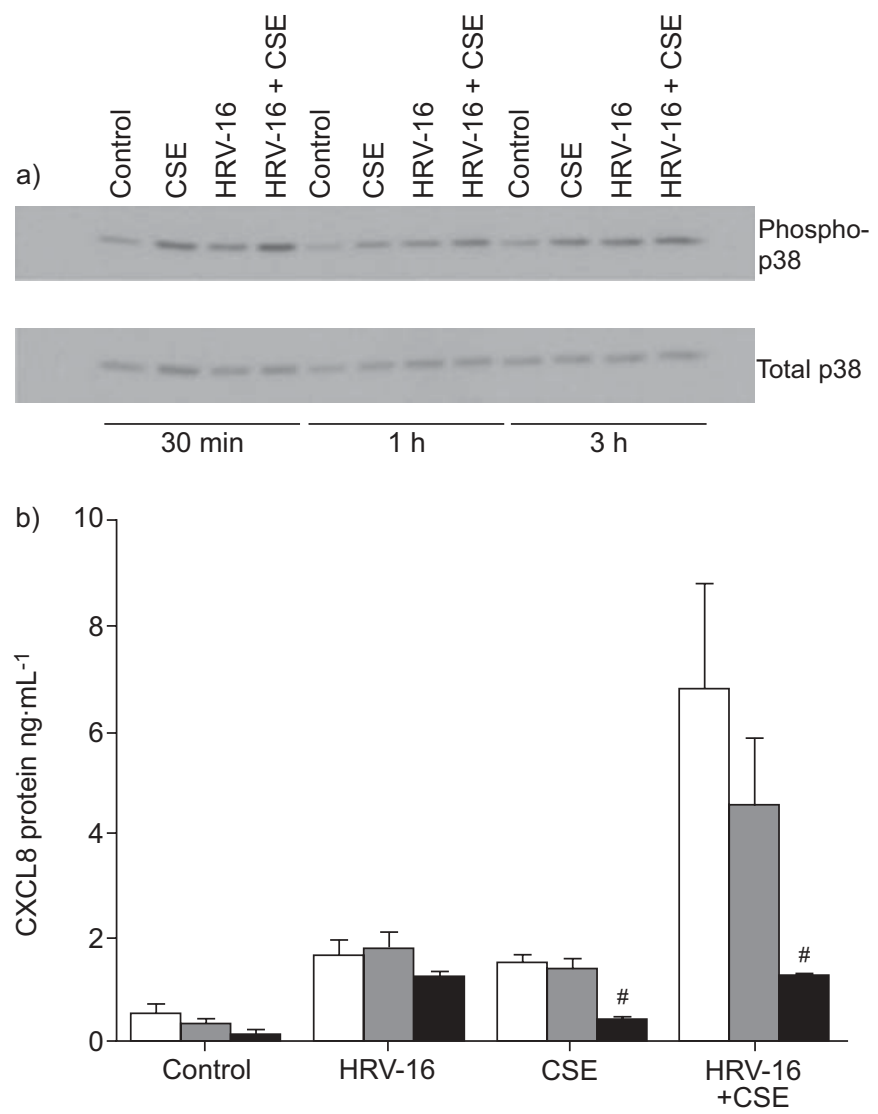

FIGURE 7. Activation of the p38 mitogen activating protein kinase (MAPK) pathway and the effects of pathway inhibition on CXC chemokine ligand (CXCL)8 production in BEAS-2B cells. a) Cells were exposed to medium, cigarette smoke extract (CSE), human rhinovirus (HRV)-16 or the combination for the times indicated and probed using a specific antibody to phospho-p38 MAPK (representative of three separate experiments). Total p38 MAPK was assayed to determine equal loading. b) Cells were incubated with stimulus alone $(\square)$ or in the presence of vehicle control (DMSO; $\mathbf{\square})$, or SB203580 (ם) for $1 \mathrm{~h}$ before stimulation with CSE, HRV-16 or the combination. \#: significant inhibition with stimulus+SB203580 compared with stimulus alone. Values are mean \pm SEM $(n=3)$.

CSE are somewhat unusual. It has been shown that CXCL10 deficient mice demonstrate increased viral replication and mortality in response to some viruses [24]. Thus cigarette smoke induced reductions in CXCL10 could impair host defences against HRV. Interestingly, mice exposed to cigarette smoke and infected with influenza show increased viral titres in their lungs compared with animals infected without smoke exposure [25]. The recent demonstration that CSE also reduces lipopolysaccaride induced epithelial production of CXCL10 [26], raises the possibility that host defences against bacterial infections could also be impaired by cigarette smoke.

The ability of CSE to modulate HRV-16-induced epithelial chemokine production does not appear to be mediated by unstable, volatile components of CSE, as very comparable results were observed using CSE prepared $24 \mathrm{~h}$ prior to use. Interestingly, CSE was markedly more potent in suppressing viral induction of CXCL10 than in inducing or enhancing CXCL8 production, suggesting that different mechanisms may 
be involved. It is known that CXCL8 can be induced in epithelial cells by HRV-16 in a manner that does not require viral replication but involves direct signalling via intracellular adhesion molecule (ICAM)-1 [27]. By contrast, HRV-induced CXCL10 production is absolutely dependent upon viral replication [4]. Nonetheless, suppression of epithelial CXCL10 production by CSE is not mediated by effects on viral replication. Not only does CSE not alter viral titres from infected cells, but induction of CXCL10 by synthetic dsRNA is also inhibited by CSE. The effects of CSE are mediated, at least in part, at the level of transcription since CXCL10 promoter activation by both HRV-16 infection and dsRNA exposure is inhibited by CSE. Interestingly, basal activation of the CXCL10 promoter was also inhibited in the presence of CSE. This suggests that CSE either induces repressive factors that suppress both basal and HRV-induced transcription, inhibits responses triggered by constitutively active transcription factors, or that CSE directly impacts the basal transcriptional complex. Further studies are needed to delineate the mechanisms of transcriptional repression of CXCL10 by CSE.

Although CSE enhanced epithelial expression of both CXCL8 mRNA and protein, it did not induce CXCL8 promoter activation and, indeed, inhibited HRV-16-induced CXCL8 promoter drive, despite the more than additive induction of steady state CXCL8 mRNA. This indicated that transcriptional regulation did not underlie the more than synergistic induction of CXCL8 by HRV-16 and CSE. It is well established, however, that expression of CXCL8 is also regulated at the level of mRNA stability, and it has been shown that activation of the p38 MAPK pathway leads to stabilisation of CXCL8 mRNA [19]. We demonstrated that treatment with the combination of HRV-16 and CSE led to a marked stabilisation in CXCL8 mRNA compared with either stimulus alone. Both stimuli induced phosphorylation of p38 and, at the earliest time point, this induction by the combination of stimuli was additive. The fact that induction by the combination of HRV-16 and CSE became less than additive at later time points when mRNA was still stable raises questions about whether p38 is the sole mechanism regulating mRNA stability. Nonetheless, selective inhibition of the $\mathrm{p} 38$ pathway reduced CXCL8 production by each stimulus alone, with significant effects on CSE and particularly on CXCL8 production by the combination of HRV and CSE, clearly indicating that this pathway plays a role in the synergistic induction of CXCL8 induction by these stimuli.

In summary, we demonstrate for the first time that CSE can modulate HRV-induced chemokine production from airway epithelial cells. CSE in combination with HRV-16 leads to an at least additive induction of CXCL8 and this synergy is mediated, at least in part, at the level of mRNA stabilisation, with some role for the p38 MAPK pathway. CSE is more potent in suppressing HRV-16-induced production of CXCL10 and this effect is mediated, at least in part, at the level of transcriptional control. Additional studies are needed to further delineate the mechanisms by which CSE modulates viral induction of these chemokines. If cigarette smoke modulates viral responses in the same way in vivo, this would suggest that patients who smoke would have increased neutrophilia and decreased recruitment of lymphocytes populations compared with nonsmokers. This change in inflammatory pattern could lead to worse exacerbations of lower airway diseases, such as COPD and asthma.

\section{SUPPORT STATEMENT}

The present study was supported by funding from the Canadian Institutes of Health Research (grant number 43923; Ottawa, ON, Canada) and by a grant from the Lung Association of Alberta and Northwest Territories (Edmonton, AB, Canada). D. Proud is the holder of a Canada Research Chair in Inflammatory Airway Diseases. M. Hudy is the recipient of a studentship from the Lung Association of Alberta and Northwest Territories.

\section{STATEMENT OF INTEREST}

None declared.

\section{REFERENCES}

1 Traves SL, Proud D. Viral-associated exacerbations of asthma and COPD. Curr Opin Pharmacol 2007; 7: 252-258.

2 Proud D, Chow C-W. Role of viral infections in asthma and chronic obstructive pulmonary disease. Am J Respir Cell Mol Biol 2006; 35: 513-518.

3 Turner $\mathrm{RB}$, Weingand $\mathrm{KW}$, Yeh $\mathrm{CH}$, et al. Association between interleukin-8 concentration in nasal secretions and severity of symptoms of experimental rhinovirus colds. Clin Infect Dis 1998; 26: $840-846$.

4 Spurrell JCL, Wiehler S, Zaheer RS, et al. Human airway epithelial cells produce IP-10 (CXCL10) in vitro and in vivo upon rhinovirus infection. Am J Physiol Lung Cell Mol Physiol 2005; 289: L85-L95.

5 Keatings VM, Collins PD, Scott DM, et al. Differences in interleukin-8 and tumor necrosis factor-alpha in induced sputum from patients with chronic obstructive pulmonary disease or asthma. Am J Respir Crit Care Med 1996; 153: 530-534.

6 Wark PAB, Johnston SL, Moric I, et al. Neutrophil degranulation and cell lysis is associated with clinical severity in virus-induced asthma. Eur Respir J 2002; 19: 68-75.

7 Barnes PJ. Chronic obstructive pulmonary disease. $N$ Engl J Med 2000; 343: 269-280.

8 Thomson NC, Chaudhuri R, Livingston E. Asthma and cigarette smoking. Eur Respir J 2004; 24: 822-833.

9 Althuis MD, Sexton M, Prybylski D. Cigarette smoking and asthma symptom severity among adult asthmatics. J Asthma 1999; 36: 257-264.

10 Chalmers GW, Macleod KJ, Little SA, et al. Influence of cigarette smoking on inhaled corticosteroid treatment in mild asthma. Thorax 2002; 57: 226-230.

11 Blake GH, Abell TD, Stanley WG. Cigarette smoking and upper respiratory infection among recruits in basic combat training. Ann Intern Med 1988; 109: 198-202.

12 Cohen S, Tyrrell DA, Russell MA, et al. Smoking, alcohol consumption, and susceptibility to the common cold. Am J Public Health 1993; 83: 1277-1283.

13 Sanders SP, Siekierski ES, Porter JD, et al. Nitric oxide inhibits rhinovirus-induced cytokine production and viral replication in a human respiratory epithelial cell line. J Virol 1998; 72: 934-942.

14 Churchill L, Chilton FH, Resau JH, et al. Cyclooxygenase metabolism of endogenous arachidonic acid by cultured human tracheal epithelial cells. Am Rev Respir Dis 1989; 140: 449-459.

15 Wirtz HRW, Schmidt M. Acute influence of cigarette smoke on secretion of pulmonary surfactant in rat alveolar type II cells in culture. Eur Respir J 1996; 9: 24-32.

16 Mosser AG, Brockman-Schneider R, Amineva S, et al. Similar frequency of rhinovirus-infectable cells in upper and lower airway epithelium. J Infect Dis 2002; 185: 734-743. 
17 Wiehler S, Proud D. Interleukin-17A modulates human airway epithelial responses to human rhinovirus infection. Am J Physiol Cell Mol Physiol 2007; 293: L505-L515.

18 Koetzler R, Zaheer RS, Wiehler S, et al. Nitric oxide inhibits human rhinovirus-induced transcriptional activation of CXCL10 in airway epithelial cells. J Allergy Clin Immunol 2009; 123: 201-208.

19 Hoffmann E, Dittrich-Breiholz O, Holtmann H, et al. Multiple control of IL-8 gene expression. J Leukocyte Biol 2002; 72: 847-855.

20 Beisswenger C, Platz J, Seifart C, et al. Exposure of differentiated airway epithelial cells to volatile smoke in vitro. Respiration 2004; 71: 402-409.

21 Mio $\mathrm{T}$, Romberger $\mathrm{DJ}$, Thompson $\mathrm{AB}$, et al. Cigarette smoke induces interleukin-8 release from human bronchial epithelial cells. Am J Respir Crit Care Med 1997; 155: 1770-1776.

22 Wark PAB, Bucchieri F, Johnston SL, et al. IFN- $\gamma$-induced protein 10 is a novel biomarker of rhinovirus induces asthma exacerbation. J Allergy Clin Immunol 2007; 120: 586-593.
23 Edwards MR, Johnson MW, Johnston SL. Combination therapy. Synergistic suppression of virus-induced chemokines in airway epithelial cells. Am J Respir Cell Mol Biol 2006; 34: 616-624.

24 Dufour JM, Dziejman M, Liu MT, et al. IFN- $\gamma$-inducible protein 10 (IP-10; CXCL10)-deficient mice reveal a role for immunoprecipitation-10 in effector T-cell generation and trafficking. J Immunol 2002; 168: 3915-3204.

25 Gualano RC, Hansen MJ, Vlahos R, et al. Cigarette smoke worsens lung inflammation and impairs resolution of influenza infection in mice. Respir Res 2008; 9: 53.

26 Pace E, Ferraro M, Siena L, et al. Cigarette smoke increases Tolllike receptor 4 and modifies lipopolysaccharide-mediated responses in airway epithelial cells. Immunology 2008; 124: 401-411.

27 Wang X, Lau C, Wiehler S, et al. Syk is downstream of intercellular adhesion molecule- 1 and mediates human rhinovirus activation of p38 MAPK in airway epithelial cells. J Immunol 2006; 177: 6859-6870. 